\title{
CARACTERIZACIÓN QUÍMICA DEL ACEITE OBTENIDO POR MÉTODOS ARTESANALES DE TRES VARIEDADES DE PALMA AFRICANA (Elaeis guineensis Jacq.)
}

\section{CHEMICAL CHARACTERIZATION OF OIL OBTAINED BY HOMEMADE METHODS FROM THREE AFRICAN PALM VARIETIES (Elaeis guineensis Jacq.)}

\author{
Ana-María Sandoval-García ${ }^{1}$ J. Reyes Altamirano-Cárdenas*, \\ Jorge Aguilar-Ávila ${ }^{2}$ y José G. García-Muñiz ${ }^{3}$
}

\begin{abstract}
'Departamento de Ingeniería Agroindustrial, Universidad Autónoma Chapingo. Km 38.5 Carr. México-Texcoco, 56230. Chapingo, Estado de México. ${ }^{2}$ Centro de Investigaciones Económicas, Sociales y Tecnológicas de la Agroindustria y la Agricultura Mundial (CIESTAAM), Universidad Autónoma Chapingo. Km 38.5 Carr. México-Texcoco, 56230. Chapingo, Estado de México. ${ }^{3}$ Posgrado en Producción Animal, Universidad Autónoma Chapingo. Km 38.5 Carr. México-Texcoco, 56230. Chapingo, Estado de México.
\end{abstract}

*Autor de correspondencia (reyesalca@yahoo.com)

RESUMEN

El cultivo de palma africana (Elaeis guineensis Jacq.) y la producción industrial de aceite han cobrado importancia en el sureste mexicano donde los productores extraen aceite artesanalmente para consumo familiar. El objetivo del estudio fue caracterizar el aceite obtenido artesanalmente de tres variedades de palma africana: Ghana, Ekona y Nigeria, en huertos de pequeños productores de Tzeltal Mukul Ja, Chiapas, México. Se determinó el perfil de ácidos grasos y se cuantificaron las vitaminas $A, D$ y E, en cada muestra de cinco parcelas y de un aceite industrializado. No se encontraron diferencias significativas en el contenido de ácidos grasos entre las tres variedades ni de vitaminas A, D y E. En general, las concentraciones de ácidos grasos y vitaminas de los aceites artesanales fueron similares a los del aceite de palma industrializado, excepto por una reducción significativa en el contenido ácido linoleico y mayor contenido de vitamina $\mathrm{A}$ en el aceite industrial. En conclusión, los aceites obtenidos artesanalmente son buena fuente de ácidos grasos y vitaminas liposolubles para el consumo humano.

Palabras clave: Elaeis guineensis, aceite de palma, palma de aceite, ácidos grasos, vitaminas liposolubles.

\section{SUMMARY}

Oil palm (Elaeis guineensis Jacq.) cultivation has become important in southeastern México where producers extract oil from part of the production for family consumption. The aim of the study was to characterize the oil obtained by artisanal processing of three palm varieties of palm: Ghana, Nigeria and Ekona grown by small producers at Tzeltal Mukul Ja, Chiapas. The fatty acid profile was determined and vitamins A, D and $E$ were measured in each sample of five orchards and from an industrial oil. No significant differences in the fatty acid content among the three varieties were found. The content of vitamins $A, D$ and $E$ showed no significant differences between varieties. In general, concentrations of fatty acids and vitamins of artisanal oils were similar to those of oil industrialized palm, except for a significant reduction in linoleic acid content and higher content of vitamin A in the industrial oil. Indeed the oils obtained using artisanal processing are a good source of fatty acids and fat-soluble vitamins for human consumption.

Index words: Elaeis guineensis, palm oil, oil palm, fatty acids, fatsoluble vitamins.

\section{INTRODUCCIÓN}

Debido a su potencial productivo el cultivo de la palma de aceite (Elaeis guineensis Jacq.) se ha extendido en el mundo, convirtiéndose en el segundo tipo de aceite con mayor volumen producido. En México, su producción se concentra en los estados del sureste, con una superficie total cosechada de 61,548 ha y 462,662 t de producción al año. Chiapas es el principal estado productor con $70.3 \%$ del total nacional, seguido de Tabasco (14.3\%) y Veracruz (10.3\%) (SIAP-SAGARPA, 2012).

Los pobladores de la comunidad de Tzeltal Mukul Ja en el municipio de Palenque, Chiapas, se dedican a la producción de palma africana, cosechan el fruto, y lo venden a dos empresas. Estas empresas, extraen el aceite crudo y lo envían a otras entidades de la república, donde se refina. El producto refinado se usa principalmente en la industria alimentaria, tanto en frituras como añadido a diversos alimentos de consumo directo. Así mismo, se usa para suplementar dietas de rumiantes en crecimiento (Partida et al., 2007). También se usa el aceite de palma en la industria cosmética para preparar jabones, dentífricos y cremas; incluso, puede ser materia prima en la obtención de biodiesel.

Los productores de Tzeltal Mukul Ja destinan parte de su producción a la elaboración de aceite para autoconsumo mediante una técnica de extracción artesanal. El proceso de extracción realizado por los productores es de menor eficiencia que el realizado por la agroindustria ya que en este último se obtienen rendimientos de alrededor de 2.3 $\mathrm{L}$ por $10 \mathrm{~kg}$ de fruta mientras que en el proceso artesanal apenas se obtiene $1 \mathrm{~L}$ de aceite por cada 7 a $10 \mathrm{~kg}$ de fruta. 
Del proceso artesanal se extraen dos clases de aceite, los cuales se clasifican como "de primera" y "de segunda". El primero tiene las mejores características para consumo humano en crudo y para su uso en alimentación animal como lo mencionan varios autores (Baucells, 2011; Ojeda y Escobar, 1995). El aceite "de segunda" se obtiene de un segundo proceso aplicado al subproducto de la primera extracción y puede ser empleado en la alimentación de aves y porcinos, lo que representa un beneficio extra para los productores.

El consumo del aceite obtenido en las unidades de producción significa un ahorro para los productores, pero se desconoce su composición y es importante estudiarla ya que puede ser una buena fuente de energía y de vitaminas liposolubles A, D y E. Por esto, el objetivo del estudio fue caracterizar químicamente el aceite de las tres variedades de palma que se producen en una comunidad, así como el aceite procesado por la agroindustria a fin de realizar un análisis comparativo.

\section{MATERIALES Y MÉTODOS}

El estudio se realizó con pequeños palmicultores en la comunidad de Tzeltal Mukul Ja, ubicada en el municipio de Palenque en el estado de Chiapas, México. La comunidad está situada a 20 msnm, a $17^{\circ} 36^{\prime} 42^{\prime \prime} \mathrm{N}$ y $92^{\circ} 06^{\prime} 41^{\prime \prime} \mathrm{O}$. El clima es cálido húmedo, con temperatura media anual de $26^{\circ} \mathrm{C}$, precipitación pluvial de $2762 \mathrm{~mm}$ al año, con vientos dominantes que provienen del norte, especialmente en el invierno. En esta zona, los pobladores se dedican principalmente al cultivo de palma africana y en algunos casos a la ganadería.

\section{Colecta, extracción y muestreo de aceites}

Se utilizaron frutos de tres variedades de palma africa- na: Deli x Ekona, Deli x Ghana y Deli x Nigeria (Figura 1), que fueron cosechados de cinco parcelas que cuentan con sistema de riego, con las tres variedades mencionadas, y por la disponibilidad de los propietarios para efectuar la extracción el aceite de manera artesanal para esta investigación.

Se recolectaron de dos a tres frutos de cada variedad en cada una de las cinco parcelas. Los frutos cosechados fueron seleccionados por los productores conforme a su propio método visual para estimar la madurez de la cosecha comercial. Cada productor llevó a cabo la extracción de aceite de acuerdo con el método artesanal que habitualmente emplea, que en todos los casos consistió en prensado manual y separación (Figura 2).

En cada huerto se tomaron cinco muestras que corresponden a 15 muestras por parcela. Además, se obtuvo una muestra de aceite de una agroindustria local (cinco repeticiones), que corresponde a la mezcla de las tres variedades de palma. En total se colectaron 80 muestras de aceite depositadas en envases de plástico ámbar, para evitar mayor exposición a la luz cuyo traslado se hizo en frío a los laboratorios de análisis.

\section{Métodos de análisis de los aceites}

Determinación de humedad. Para analizar el contenido de agua en el aceite se empleó el secado en estufa, técnica que se basa en la pérdida de peso de la muestra por evaporación del agua. Para esto se requiere que la muestra sea térmicamente estable y que no contenga una cantidad significativa de compuestos volátiles. El principio operacional incluye la preparación de la muestra, consistente en pesado, secado, enfriado y pesado nuevamente de la muestra de acuerdo con el método descrito en el Apartado 926.12 de la AOAC (1990).

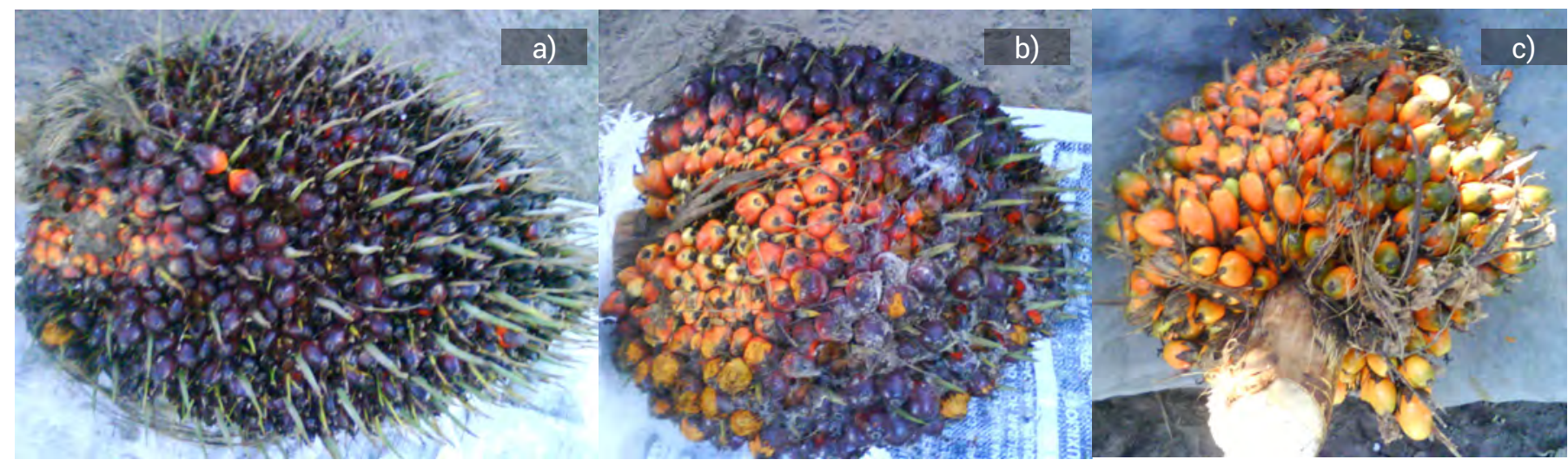

Figura 1. Frutos de las variedades de palma africana empleados en el estudio: a) Deli x Ekona, b) Deli x Ghana, y c) Deli $x$ Nigeria. 


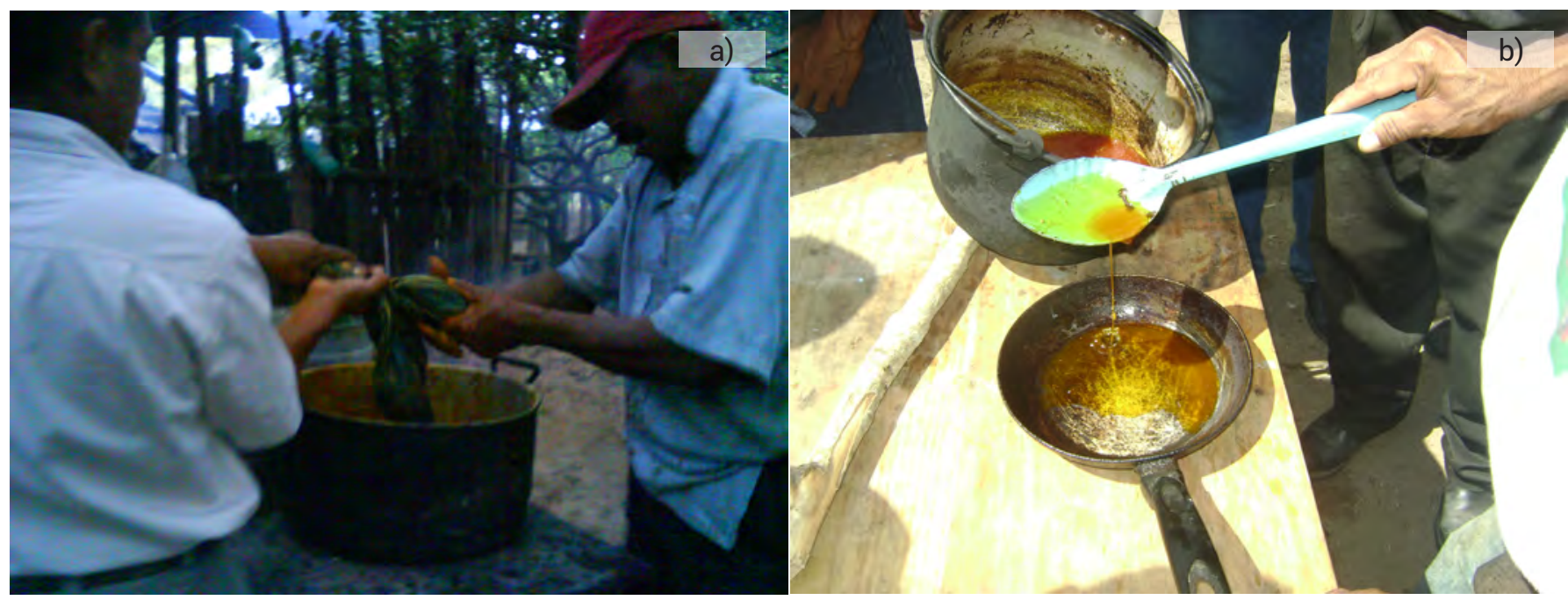

Figura 2. Imágenes que ilustran parte del proceso de extracción artesanal del aceite: a) prensado manual, b) separación del aceite.

Análisis de ácidos grasos. Las muestras fueron saponificadas y metiladas de acuerdo con lo descrito por Murrieta et al. (2003), con algunas modificaciones en la metodología. Para determinar el contenido de ácidos grasos se prepararon las muestras de $1.5 \mathrm{~mL}$ de aceite extraídas del análisis de materia seca, que se guardaron en viales para su posterior tratamiento. De cada muestra de aceite se pesaron $0.0050 \mathrm{~g}$ y se les adicionó $1 \mathrm{~mL}$ de hexano; de esta mezcla se tomaron $10 \mu \mathrm{L}$ y se colocaron en un vial, para posteriormente agregar $40 \mu \mathrm{L}$ de hidróxido de trimetil sulfonium (HTS).

La nueva mezcla se agitó y se mantuvo en refrigeración por $2 \mathrm{~h}$ para permitir la metilación de los ácidos. Transcurrido este tiempo se inyectó $1 \mu \mathrm{L}$ en el cromatógrafo de gases marca Perkin Elmer ${ }^{\circledR}$ Varian auto-System equipado con un detector y un integrador de flama ionizante, con una columna capilar de $30 \mathrm{~m} \times 225 \mathrm{~mm} \times 0.225 \mathrm{~mm}$. La temperatura del inyector se estabilizó a partir de $130^{\circ} \mathrm{C}$ y se aumentó $1^{\circ} \mathrm{C}$ a la vez, hasta alcanzar $150{ }^{\circ} \mathrm{C}$; la temperatura de la columna se estableció en $200{ }^{\circ} \mathrm{C}$ y la del detector se estableció en $250^{\circ} \mathrm{C}$

Para el acarreo de la muestra se empleó una mezcla de gases marca Infra ${ }^{\circledR}$ constituida por $\mathrm{H}, \mathrm{He}$ y aire comprimido a 1,378.95:551.58:1,378.95 kPa cm-2 por "split" (dispositivo para diluir) a un flujo de $1 \mathrm{~mL} \mathrm{~min}^{-1}$. Los ácidos grasos se identificaron por comparación con los tiempos de retención de los respectivos estándares de los ácidos grasos usados en el análisis, todos de la marca Sigma® (99\% de pureza). Los estándares se procesaron bajo las mismas condiciones que las muestras y se inyectaron con tres repeticiones cada uno para establecer sus tiempos de retención.
Análisis de vitaminas liposolubles. La extracción de las vitaminas se llevó a cabo de acuerdo con la metodología descrita por Macrae (1998), modificada. Se pesó $1 \mathrm{~g}$ de aceite y se adicionaron $35 \mathrm{~mL}$ de etanol absoluto, $10 \mathrm{~mL}$ de $\mathrm{KOH}$ al $50 \%$ y $25 \mathrm{mg}$ de hidroquinona como antioxidante; se calentó la mezcla a reflujo durante 30 min a temperatura no mayor de $35^{\circ} \mathrm{C}$ con agitaciones periódicas; la mezcla se retiró del calor y se agregaron $40 \mathrm{~mL}$ de agua destilada y $50 \mathrm{~mL}$ de una mezcla éter dietílico:n-hexano, se colocó en un matraz de separación donde se agitó por 5 min y se dejó reposar hasta la separación de fases. Se drenó la fase acuosa y a la fase orgánica se le realizaron cinco lavados con $50 \mathrm{~mL}$ de agua acidulada, misma a la que se le agregaron $2 \mathrm{~g}$ de $\mathrm{Na}_{2} \mathrm{SO}_{4}$.

El extracto se concentró a sequedad en un rotavapor Buchi® y se reconstituyó con $25 \mathrm{~mL}$ de $\mathrm{KOH}$ metanólico. Fue almacenado en congelación en frascos ámbar para su conservación hasta ser cuantificado en un periodo máximo de $12 \mathrm{~h}$. Todo el material fue cubierto con papel aluminio para evitar el deterioro de las vitaminas por la luz, y el aceite se mantuvo lo menos posible en contacto con el ambiente.

La identificación y cuantificación de vitamina A se realizó de acuerdo con la metodología descrita por GarcíaPeña et al. (2008) con adecuaciones, por cromatografía de líquidos de alta resolución (HPLC) en un cromatógrafo de líquidos Waters ${ }^{\circledR} 717$ autosampler (con muestreo automático), equipado con un detector UV modelo 2487 y una bomba dual. Se usó una columna Perkin Elmer ${ }^{\circledR}$ fase reversa Spheri 5 RP18 $(250 \mathrm{~mm} \times 4.6 \mathrm{~mm}$, tamaño de partículas $5 \mu \mathrm{m}$ ). La fase móvil para el caso de la vitamina A fue metanol-agua (98:2), el tiempo de corrida fue de 10 
min, la detección de la vitamina A se realizó a $325 \mathrm{~nm}$ a temperatura ambiente, con un flujo de $1.5 \mathrm{~mL} \mathrm{~min}^{-1}$. Para la identificación y cuantificación de esta vitamina se empleó como estándar a palmitato de retinol disuelto en $\mathrm{KOH}$ metanólico, para la elaboración de la curva patrón a concentraciones de 5, 10, 15, 20 y 25 ppm. Cada estándar se filtró en un acrodisco de nailon de $0.22 \mu \mathrm{m}$ Titan2® antes de ser inyectados en el HPLC.

La identificación y cuantificación de la vitamina D se llevó a cabo de acuerdo con la metodología descrita por GarcíaPeña et al. (2008) con adecuaciones, por cromatografía de líquidos de alta resolución (HPLC) en un cromatógrafo de líquidos Waters ${ }^{\circledR} 717$ autosampler, equipado con un detector UV modelo 2487 y una bomba dual. Se usó una columna Perkin Elmer ${ }^{\circledR}$ fase reversa Spheri 5 RP18 $(250 \mathrm{~mm}$ × $4.6 \mathrm{~mm}$, tamaño de partículas $5 \mu$ ). La fase móvil para el caso de la vitamina $D$ fue acetonitrilo-metanol (91:9). El tiempo de corrida fue de 20 min; la detección de la vitamina $D$ se hizo a $265 \mathrm{~nm}$ a temperatura ambiente, con un flujo de $1.5 \mathrm{~mL}$ min-1. Para la identificación y cuantificación de esta vitamina se empleó como estándar a colecalciferol disuelto en $\mathrm{KOH}$ metanólico, para la elaboración de la curva patrón a concentraciones de 5, 10, 15, 20 y 25 ppm. Cada estándar se filtró en un acrodisco de nailon de 0.22 um Titan2® antes de ser inyectados en el HPLC.

Para la identificación y cuantificación de a-tocoferol (vitamina E), se emplearon las metodologías descritas por Correa et al. (2005) y Momenbeik et al. (2005), con adecuaciones. Los tocoferoles se analizaron por cromatografía de líquidos de alta resolución (HPLC) en un equipo Waters ${ }^{\circledR}$ 717 "autosampler" equipado con un detector UV Modelo 2487 y una bomba dual. Se usó una columna Perkin Elmer ${ }^{\circledR}$ fase reversa Spheri 5 RP18 $(250 \mathrm{~mm} \times 4.6 \mathrm{~mm}$, tamaño de partículas $5 \mu$ ). La fase móvil fue metanol/agua

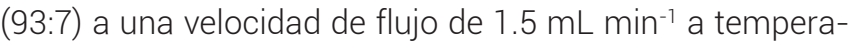
tura ambiente. La mezcla de solvente se filtró a través de un disco con poros de $0.45 \mu \mathrm{m}$ antes de usarlo, y se agitó en vacío para extraer gases disueltos.

La longitud de onda programada para a- tocoferol fue de $285 \mathrm{~nm}$ y el tiempo de corrida fue de $25 \mathrm{~min}$. Para la identificación y cuantificación de esta vitamina se empleó como estándar a-tocoferol disuelto en $\mathrm{KOH}$ metanólico, para la elaboración de la curva patrón a concentraciones de 5, 10, 15, 20 y 25 ppm. Cada estándar se filtró en un acrodisco de nailon de $0.22 \mu \mathrm{m}$ Titan2 ${ }^{\circledR}$ antes de ser inyectados en el HPLC.

\section{Métodos estadísticos}

El diseño experimental utilizado para evaluar la influencia de las distintas fuentes de variación en las variables de respuesta fue completamente al azar. Los análisis de varianza se realizaron utilizando el paquete estadístico SAS 9.0. Todos los análisis de contenido de materia seca, ácidos grasos y vitaminas fueron realizados por triplicado y la comparación de medias de los valores entre variedades se llevó a cabo con la prueba de Tukey. También se discuten las diferencias estadísticas de los valores obtenidos de ácidos grasos y vitaminas de las muestras del aceite obtenido de la agroindustría en comparación con el de la extracción artesanal utilizando para este fin, la misma prueba de Tukey.

\section{RESULTADOS Y DISCUSIÓN}

El aceite obtenido de los frutos de las parcelas P1 con las variedades Ghana y Nigeria y de la parcela P3 con las variedades Ekona y Ghana, presentaron contenidos mayores de agua con porcentajes de 11 a $35 \%$; mientras que las parcelas P4 y P5 fueron las que tuvieron menor contenido de agua (de 0.15 a $0.50 \%$ de agua) $(P<0,05)$ y un mayor porcentaje de aceite $(P<0.05)$. De acuerdo con la NMX-F019-SCFI-2006 de la Secretaría de Economía, el contenido permisible de agua para el aceite crudo de palma es de 0.5 $\%$, por lo que el aceite de las parcelas P1 y P3 están fuera de los límites; esto refleja el proceso no estandarizado que efectúan los productores en la obtención de su aceite.

Los resultados obtenidos para la muestra de aceite proveniente de la agroindustria, muestran bajos contenidos de agua es decir, con mayor contenido de aceite de 99.58 $\pm 0.029 \%$ (Cuadro 1).

En el análisis realizado al aceite artesanal se obtuvo una relación 2:2 de ácidos grasos saturados e insaturados, los cuales fueron identificados como ácido mirístico (14:0), palmítico (16:0), oleico (18:1) y linoleico (18:2). No se encontraron diferencias significativas entre parcelas ni entre variedades en las concentraciones de algunos de los ácidos grasos (Cuadro 1), y todas caen en el rango de los valores publicados para el aceite de palma (Murphy, 2014, Innis, 1991). En cuanto al aceite industrial, los contenidos de ácidos palmítico, oleico y mirístico son similares a los obtenidos del aceite artesanal; sólo hubo diferencia significativa en el contenido del ácido linoleico cuyo valor fue menor $(14.15 \pm 3.69 \%)$, lo cual puede deberse a su degradación durante el proceso de extracción (FAO/OMS, 1997).

El principal competidor en volumen de producción del aceite de palma a nivel mundial es el aceite de soya (Glycine max); al comparar sus características en contenido de ácidos grasos, el aceite crudo de palma es más rico en ácido oléico (18:1) que el aceite de soya, aunque éste contiene más ácido linoleico, mejor en calidad por ser un ácido graso poliinsaturado (FAO/OMS, 1997). 
En cuanto a las vitaminas $A$, D y E, en el aceite extraído de manera artesanal, no hubo diferencias significativas entre variedades ni entre parcelas; los valores obtenidos (Cuadro 2) se ubican en el rango de los publicados en otros estudios para este tipo de aceite (Underwood, 2000). Sin embargo, los contenidos de vitamina A de las tres variedades analizadas fueron significativamente menores $(P<0.05)$ a la media del valor obtenido en este mismo estudio para el aceite industrializado (Cuadro 2).

Los niveles encontrados para vitamina $\mathrm{E}$ coinciden con los reportados por Sáyago et al. (2007) para a-tocoferol. Las diferencias entre el aceite extraído de manera artesanal y el industrial se pueden deber a la diferencia entre procesos; en el método artesanal el aceite está expuesto al calor en presencia de $\mathrm{O}_{2}$ lo que degrada rápidamente las vitaminas, como se reporta en la literatura (Fennema, 2000), mientras que en la agroindustria la degradación es menor debido al control en el proceso.

Un aspecto interesante del aceite de palma desde el punto de vista nutricional, es la aportación de vitamina A. Por esto el aceite de palma se usa en diferentes países para el tratamiento y prevención de la avitaminosis A (Salinas et al., 2008).

\section{CONCLUSIONES}

Los aceites obtenidos artesanalmente son buena fuente de ácidos grasos y vitaminas liposolubles, para el consumo humano. El contenido de vitaminas A, D y E en las tres variedades analizadas en este estudio no presenta diferencias significativas entre variedades ni entre parcelas de productores. En general, el aceite artesanal es significativamente comparable en su contenido y calidad de ácidos grasos, entre las variedades Deli x Ekona, Deli x Ghana y Deli x Nigeria.

El aceite extraído por la agroindustria presenta contenidos similares de ácidos palmítico, oleico y mirístico que el aceite artesanal, pero contiene menos cantidad de ácido linoleico. En estos dos aceites no hay diferencias significativa en cuanto al contenido de vitaminas $D$ y E; mientras que si la hay en el contenido de vitamina A, que es mayor en el aceite industrial.

El contenido de agua fue alto en algunas muestras de aceite de las parcelas analizadas debido al proceso no estandarizado de extracción que llevan a cabo los productores de la comunidad. El aceite obtenido de la planta agroindustrial en comparación con el extraído con productores de la comunidad de manera artesanal, tiene un menor contenido de agua.

\section{AGRADECIMIENTOS}

Este trabajo fue financiado por la Dirección General de Investigación y Posgrado (DGIP) de la Universidad Autónoma

Cuadro 1. Porcentaje de ácidos grasos y materia seca en muestras de aceite artesanal e industrial obtenido de variedades de palma africana.

\begin{tabular}{lcccc}
\hline \multirow{2}{*}{ Componente } & \multicolumn{4}{c}{ Variedad de palma de aceite } \\
\cline { 2 - 5 } & Deli x Ekona & Deli x Ghana & Deli x Nigeria & Mezcla industrial \\
\hline MS (\%) & $88.15 \pm 14.33 \mathrm{a}$ & $90.79 \pm 9.42 \mathrm{a}$ & $92.05 \pm 7.52 \mathrm{a}$ & $99.58 \pm 0.029 \mathrm{~b}$ \\
Ac. Palmítico (\%) & $33.10 \pm 5.13 \mathrm{a}$ & $35.48 \pm 3.82 \mathrm{a}$ & $34.25 \pm 3.43 \mathrm{a}$ & $34.29 \pm 1.47 \mathrm{a}$ \\
Ac. Oleico (\%) & $43.31 \pm 5.09 \mathrm{a}$ & $42.21 \pm 3.51 \mathrm{a}$ & $43.02 \pm 3.31 \mathrm{a}$ & $46.96 \pm 4.48 \mathrm{a}$ \\
Ac. Linoleico (\%) & $20.31 \pm 3.08 \mathrm{a}$ & $18.56 \pm 3.75 \mathrm{a}$ & $18.93 \pm 3.59 \mathrm{a}$ & $14.15 \pm 3.69 \mathrm{~b}$ \\
Ac. Mirístico (\%) & $2.08 \pm 1.09 \mathrm{a}$ & $1.76 \pm 0.48 \mathrm{a}$ & $1.80 \pm 1.04 \mathrm{a}$ & $2.04 \pm 0.69 \mathrm{a}$ \\
\hline
\end{tabular}

MS = materia seca; Medias con letras iguales en cada fila no son estadísticamente diferentes (Tukey, 0.05).

Cuadro 2. Contenido de vitaminas liposolubles $\left(\mathrm{mg} \mathrm{mL}^{-1}\right)$ en el aceite de tres variedades de palma africana y con dos métodos de extracción.

\begin{tabular}{lcccc}
\hline \multirow{2}{*}{ Componente } & \multicolumn{4}{c}{ Variedad de palma de aceite } \\
\cline { 2 - 5 } & Deli x Ekona & Deli $\times$ Ghana & Deli $\times$ Nigeria & Mezcla Industrial \\
\hline Vitamina A & $15.39 \pm 5.99 \mathrm{a}$ & $16.32 \pm 2.00 \mathrm{a}$ & $15.17 \pm 1.87 \mathrm{a}$ & $33.66 \pm 10.91 \mathrm{~b}$ \\
Vitamina D & $42.17 \pm 3.52 \mathrm{a}$ & $43.65 \pm 8.18 \mathrm{a}$ & $45.54 \pm 6.89 \mathrm{a}$ & $47.89 \pm 2.08 \mathrm{a}$ \\
Vitamina E & $41.60 \pm 10.76 \mathrm{a}$ & $26.71 \pm 8.33 \mathrm{a}$ & $36.80 \pm 5.99 \mathrm{a}$ & $35.76 \pm 8.07 \mathrm{a}$ \\
\hline
\end{tabular}

Medias con letras iguales en cada fila no son estadísticamente diferentes (Tukey, 0.05). 
Chapingo como parte de un proyecto de investigación. Los autores agradecen la participación de los palmicultores de la comunidad de Tzeltal Mukul Ja, municipio de Palenque, Chiapas y al laboratorio de nutrición de rumiantes de la Facultad de Medicina Veterinaria y Zootecnia de la Universidad Autónoma del Estado de México, por el préstamo y la asistencia técnica en el uso del HPLC para la cuantificación de vitaminas.

\section{BIBLIOGRAFÍA}

AOAC (1990) Official Methods of Analysis 15th ed. Association of Official Analytical Chemists. Washington

Baucells J. (2011) El aceite de palma es una buena alternativa en la alimentación de rumiantes. Albéitar 130:10-12.

Correa S., O. Busto and J. Guasch (2005) Simultaneous Quantification of Phytosterols and Tocopherols in Almond Oil by Reversed-phase High-Performance Liquid Chromatograpy. Grup de Química Analítica Enològica i dels Aliments (Unitat d' Enologia del CeRTA), Departament de Química Analítica i Química Orgánica Facultat d'Enologia, Universitat Rovira i Virgili, Terragona, Spaña. pp:6-36.

FAO/OMS (1997) Grasas y Aceites en la Nutrición Humana. Estudio FAO, Alimentación y Nutrición 57. Organización de las Naciones Unidas para la Agricultura y la Alimentación. Roma. http://www. fao.org/docrep/v4700s/v4700s00.html (Agosto 2014).

Fennema R. O. (2000) Química de los Alimentos. $2^{a}$ ed. Ed. Acribia S.A. $1258 p$

García-Peña C. M., M. Castiñeira Díaz, M. Fernández Cervera, J. A. Romero Díaz, S. M. Collazo Quintana, B. Mancebo Dorvignny y L. Torres Amaro (2008) Determinación por CLAR de las vitaminas A y D en aceite de hígado de tiburón. Latin American Journal of Pharmacy 27:419-24.

Innis M. (1991) Essential fatty acids in growth and development. Progress in Lipid Research 30:39-103.
Lawson H. W. (1985) Standard for Fats and Oils. The L. J. Minor Food Service Standards Series, Volume 5. The AVI Publishing Company Inc. Connecticut, USA.

Macrae A. R. (1988) HPLC in Food Analysis. 2a ed. Academic Press, Inc. London, Great Britain. 502 p.

Momenbeik F., Z. Momeni and J. H. Khorasani (2005) Separation and determination of vitamins $E$ and $A$ in multivitamin syrup using micellar liquid chromatography and simplex optimization. Journal of Pharmaceutical and Biomedical Analysis 37:383-387.

Murphy D. J. (2014) The future of oil palm as a major global crop: opportunities and challenges. Journal of Oil Palm Research 26:1-24.

Murrieta C. M., B. W. Hess and D. C. Rule (2003) Comparison of acidic and alkaline catalysts for preparation of fatty acid methyl esters from ovine muscle with emphasis on conjugated linoleic acid Meat Science 65:523-529.

Ojeda, A. y A. Escobar (1995) Suplementación con aceite crudo de palma africana de bovinos para ceba en pastoreo. CIPAV. Livestock Research for Rural Development 7:1-8.

Partida J. A., J. L. Olleta, C. Sañudo, P. Alberti and Campo M. M. (2007) Fatty acid composition and sensory traits of beef fed palm oil supplements. Meat Science 76:444-454.

Salinas N., M. Márquez, R. Sutil, E. Pacheco, M. Muñoz y M. E. Gómez (2008) Evaluación del efecto de un aceite de palma parcialmente refinado con un alto contenido en micronutrientes sobre el perfil lipídico de ratas. Investigación Clínica 49:5-16.

Sáyago A., M. I. Marín, R. M. Aparicio y T. Morales (2007) Vitamina E y aceites vegetales. Grasas y Aceites 58:74-86.

SE, Secretaría de Economía (2012) Norma Mexicana NMX-F-019-SCF -2012. Alimentos-Aceite de palma-especificaciones. Secretaría de Economía. Diario Oficial de la Federación, Jueves 10 de mayo de 2012 (Junio 2012)

SIAP-SAGARPA (2012) Estadísticas de producción anual de palma africana. Sistema de Información Agroalimentaria y Pesquera. http://www.siap.sagarpa.gob.mx/ (Junio 2012)

Underwood B. A. (2000) Dietary approaches to the control of vitamin A deficiency: An introduction and overview. Food and Nutrition Bulletin 21:117-123.

Wan P. J. (2000) Properties of fats and oils. In: Introduction to Fats and Oils Technology. R. D. O'Brien, W. E. Farr and P. J. Wan (eds.) AOCS Press. Illinois, U. S. A. pp:20-48. 